bruć, welder anfangs auf bie balfamifde Rinbe yon Myroxylon anjewanot wurbe, Ponate auf ble ber Eindonen iibertragen werben, bie burd igre fiebervertreibenbe Rraft fo widtig tourben. Ilus berfelben Urfade fieft obne Zweifel alld die Anjīt, baj die beffern Ebinarinden aum barjigfen erfdeinen unb einen balfamifden (jerud aubgeben múffen.

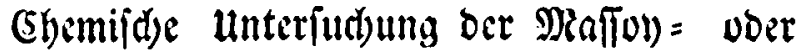 $\mathfrak{R} \mathfrak{a}_{j} \mathbf{o i}=\mathfrak{R i n d e}$; oon \\ Sh. Bonaftre*).
}

Der Baum, welder biefe Rinde liefett, wadft auf Rell s Guinea uno farint ju ber Familie ber Laurineen uno jur Battung Laurus L. ju geţüren. Die hellgrúnen, wes nig glänjenden oblongen Blatter baben einen rebre aromatis f由en Berud. Die unterfudte Ritroen, welde elger von Aeften als yom Stamme berjuríbren foienen, waren aus, Bertid weifflidt ober leidt gelblidts grau, uno mit mehres ren Eryptogamen, wie Porina marginata (Fée), bebedt, ber Brud wat uneben, bas Bervebe fdrwammig, etras diå uno felin zerbredlid. Im Junern war fie fahlgetb,

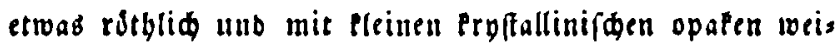

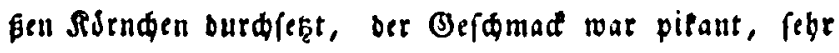
wenig bitter unb reijte ben Epeidel; bet farte Oerud war vem bes Eaffafras ábulid.

\section{2t nalyre.}

25 Grammen der Maffoy = Minde gaben ourd Deffilo

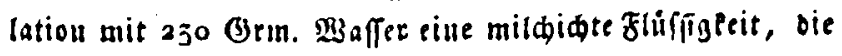

*) Journ. de Pharm. XV. 200. 93. B r. 
orei verf由iebene Probufte entbielt; ein dtberifdes Del,

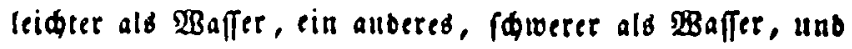
eine pulverfơrmige, balb concretirte Subftan\}, eben to folver als Baffer.

Das leidte diberifकe Del ift befonders aromatifí, wie bas deberifde Saffafrabst; es ift faft ungefarbt; bat cinen farten, febr pituuten, fpeidelerregenden Gefdmad; IJft fid villig in 2lttobol, Edivefelatber uno felbft in concen,

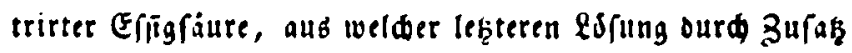
von orei Theilen $28 a f f e r$ bas Del wieder abgefbieben wirb. Durd Salpeterfáure orbalt es bei gewishuli her Zemperatur, woie das Saliafrassl, eine bodrotbe Zarbe, welde ju einer mehe ober minber tirfarotben Favbe úbergeht. Die Mens ge oiefes Dels betrigt beinabe $\frac{x}{2} \delta$ bes erbaltenen fludtigen Probutts.

Das liwere Del ift oider, niфt fo flú⿴囗十⺝g als bas leidre Del unb finte im Baffer zu Boben, tubrigens befizt es ăhulide Eigenidaften als bie beim leidten Dele angeges benen. SRit Galpeterfare warm behandelt bilben fid Siry:

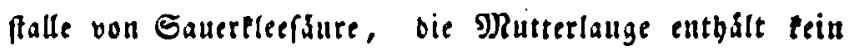
Leeltberípes Bitter. Bei ro ${ }^{\circ}$ E. wiro diefes Del trübe, et, was oid, bat alsoant aber eben fo wenig wie oas Saffafrass Sl ein Pryfallinifded Infeben. Es verbinbet fid nur rebr unvolffandig mit Rali= ober Natronlauge, mit flúfingem

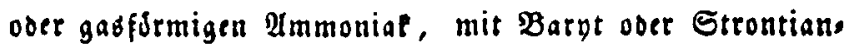
loaffer; es wiro mit biefen leşteren fart gef́luttelt wobl trúbe, weiflidt uno bid, aber biefe Berbinoung faeidet fich balo wieber.

Durd biefen lek̨teren હharacter unterfbeibet ef fí vorjiglid von bem Betwurznelfensl, von den Delen bes Pimente, myrt. piment. et caryophyllat. uno nåbert fid ben befonberen Arten ber Reibe ber Dele von Salfaftad $\mathrm{XXX} \mathfrak{8 0 .} 3 \mathrm{fgft}$. 
und ber Yidurimbobuen, bie ald ju ber Battung Laurus gebsen.

2lud Pann biefer wefentlide Ebaracter, fid mit ben Salzbafen niфt ju verbinden, daju bienen, die flidtigen Dele ber Galtang Laurus, mittelft biefer Bafen uno ber Deffillation mit Baffer, yon ben Delen ber Gattung Myrtus uno Caryophyllas zu fdeiben.

Das oritte flúdtige Prooult, weldes gegen Enve ber Deftillation übergebt, ift weiß uno pulverfsrmig. Es nass bert fid in feitlen pbyjifden uno demifden Cbaracteren bem Carpopbyllin oder vietmegr bem laurin, linterbarze, bie

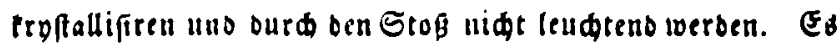

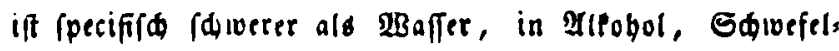
átber uno concentrirter Efingfaure Isolid; es ift faft ges ldmat, uno gerndlod; burd ealte Ealpeterfaure farbt es fid leidt gelblidt, weldeb aber wabrideinlid voin anbin: genden Dele berríbrt. Die Nenge betrágt ungefäbr 3 bis 4 Theile auf jo Theile beb flidtigen Yrodults.

Durd fernere Belyanding bed Siluffandes ber Deffillas

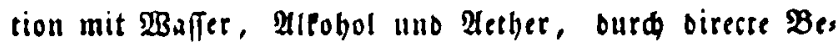
bandlung bes Rinbeupulvers mit Allpobol uno Aetber und ourd Calcination wurben erbalten: ein in taltem 2 affer Istlided foleimiges (Jumni, wenig gefseblet, tanningals tiger Extractioftoff, Startmebl, eitle nidt daracterifirte

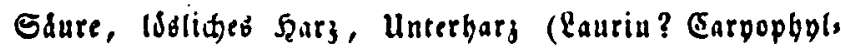
lin?), Dides butterartiges Del, ein bides bem Stearin ăg̣lided Del, Rali, und Raltfalze, uno eine febr geringe Menge $\mathfrak{F}_{\text {Zlijfafer. }}$

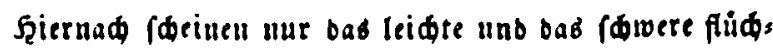
tige Del bie wirlfamen Beftandryeile ber Maffog, Rinbe zu reqn, benn alle übrigen Beftanotbeile baben nur febe wenig wirlfame Eigenfdaften. Die verfdiebenen unmittelbaren Beftanbtheile biefer Sinbe nábern fiф febr benen oon $\mathfrak{B} a \mathfrak{a}$ : 
quelin im Eeplonfden Bimmt gefunberen, rolder aud jur Gattung Laurus L. gebort.

Beitiag jur (Sefd)id)te Deg Laurus persea (adrugatolvorber, frolobillbirnbaum,

$$
\text { Arocatier); }
$$

bon

$$
\text { Rlcort = Nabianna*). }
$$

Laurus persica Linn., L. persea Jacq. gebdet ju ber

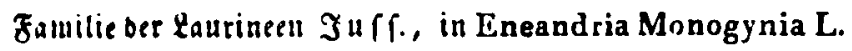
Der Baum wadff in Súdamerifa und wuroe, wie $\mathfrak{A}$ ublet erjablt, $175^{\circ}$ nad Eayeune gebradt. Nidolfon ber Bater fah auf Domingo Balume von 50 Fub $\mathfrak{B}_{\text {ghe, id }}$ felbft aber auf Guabeloupe niфt úber 30 bis 40 Juß bo Das febre brúdige weiglidete $\mathfrak{F}_{2}$ olj bient nur jum Brennen Die Friddte baben in ibrer form Aehnlideeit mit einer Duitte ober Birte, in Şinfidet ber Grope find fie verífies ben, es giebt relde von 6 3oll länge. Huf Guabeloup finoen fic nad ber Form uno Jarbe ber Frildte 6 Barietds ten: 1) mit runben und grúmen Frúdten; 2) mit runden uno violetten; 3) mit vblongen unb violetten; 4) mit obs longen uno grinen; 5) mit warjigen violetten; 6) mit warzigen grúnen $z$ rúdten.

Die F̧aut ber reifen Frudt ISft fid leidt ab; bas Fleifd ift unter biefer $\mathfrak{H}_{a}$ ult piftaciengrün unb im Innern gelblidt. Der Berdmat beffelben ift nad bem Boben unto ber mebe oder weniger regnigten Jabrebjeit, in ber man bie Frudbte einfammelt, febr verfdieben; einige fameden

*) Journ. de Pharmac. XV. 42. 84. $1+3$. 